\title{
On the magnetic properties of Gd implanted GaN
}

J. Hejtmánek, K. Knížek, M. Maryško, Z. Jirák, D. Sedmidubský, Z. Sofer, V. Peřina, H. Hardtdegen, and C. Buchal

Citation: Journal of Applied Physics 103, 07 D107 (2008);

View online: https://doi.org/10.1063/1.2830644

View Table of Contents: http://aip.scitation.org/toc/jap/103/7

Published by the American Institute of Physics

\section{Articles you may be interested in}

Stability and electronic structure of defect complexes in Gd-doped GaN: First-principles calculations Journal of Applied Physics 122, 023901 (2017); 10.1063/1.4993452

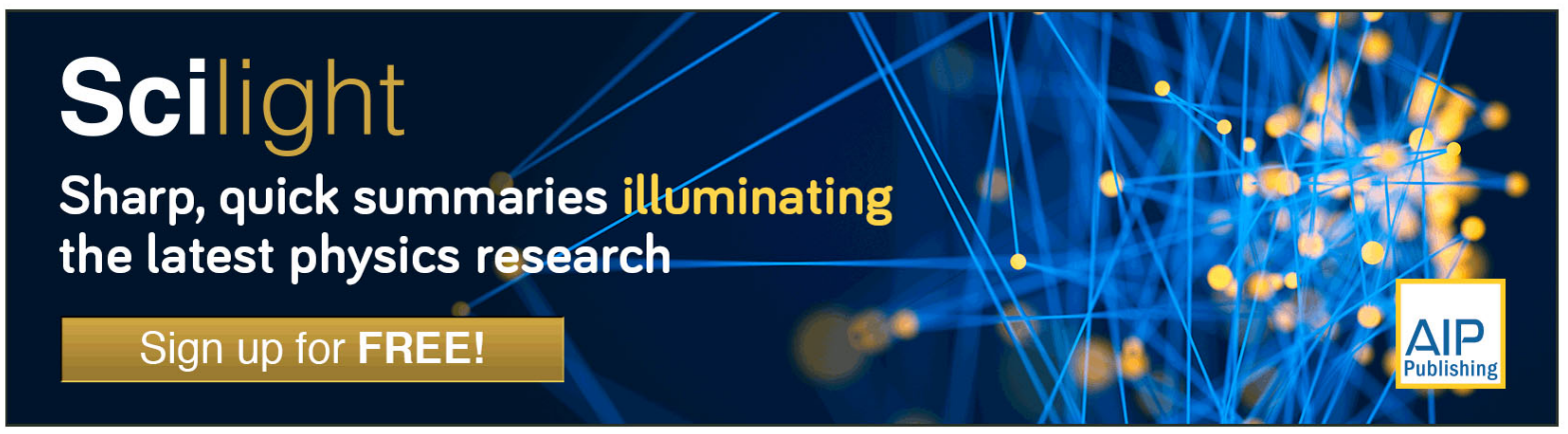




\title{
On the magnetic properties of Gd implanted GaN
}

\author{
J. Hejtmánek, ${ }^{1, a)}$ K. Knížek ${ }^{1}$ M. Maryško, ${ }^{1}$ Z. Jirák, ${ }^{1}$ D. Sedmidubský, ${ }^{2}$ Z. Sofer, ${ }^{2}$ \\ V. Peřina, ${ }^{3}$ H. Hardtdegen, ${ }^{4}$ and C. Buchal ${ }^{4}$ \\ ${ }^{1}$ Institute of Physics ASCR, Cukrovarnická 10, 16253 Prague, Czech Republic \\ ${ }^{2}$ Institute of Chemical Technology, Technická 5, 16628 Prague, Czech Republic \\ ${ }^{3}$ Nuclear Physics Institute of the ASCR, 25068 Rez, Czech Republic \\ ${ }^{4}$ Institute of Bio-Nanosystems, Forschungscentrum Jülich, 52425 Jülich, Germany
}

(Presented on 6 November 2007; received 12 September 2007; accepted 12 October 2007; published online 28 January 2008)

\begin{abstract}
The wurzite type gallium nitride doped by gadolinium, $\mathrm{Ga}_{1-x} \mathrm{Gd}_{x} \mathrm{~N}(x \sim 0.01-0.07)$, was prepared by Gd ion implantation of the parent GaN thin films deposited on sapphire substrates. The material obtained exhibits a weak ferromagnetism (FM) persisting up to $700 \mathrm{~K}$. At higher Gd concentrations, the minute FM component coexists with much more pronounced Curie-type paramagnetism. In a dilute limit $(x \leqslant 0.01)$, the latter part is substantially reduced and the saturated FM moment reaches the value $M \sim 2 \mu_{B} / \mathrm{Gd}$ atom. (C) 2008 American Institute of Physics. [DOI: 10.1063/1.2830644]
\end{abstract}

\section{INTRODUCTION}

The practical development of the novel spin-based devices depends on the availability of materials with magnetic ordering $\left(T_{C}\right)$ above room temperature. ${ }^{1}$ Most recently a weak ferromagnetism with extremely high values of $T_{C}$ $\sim 700 \mathrm{~K}$ was reported in $\mathrm{Ga}_{1-x} \mathrm{Gd}_{x} \mathrm{~N}$ films, prepared either by the molecular beam epitaxy (MBE) technique or by Gd ion implantation into pure $\mathrm{GaN}^{2-5}$ Moreover this exotic ferromagnetism is apparently accompanied, namely, for low $\mathrm{Gd}$ concentrations, by a giant magnetic moment reportedly up to $10^{4} \mu_{B} / \mathrm{Gd}$ ion. ${ }^{2}$ In this work, we thus present a magnetic study of Gd implantation into GaN films grown by low pressure metal-organic vapor phase epitaxy (MOVPE) on $c$-plane sapphire substrates. Compared to the previous reports on analogous Gd implantation, substantially higher doses have been used and the films were additionally annealed in order to reduce the implantation damage and to recover the crystal structure. The doped films were characterized by a steep depth profile of the Gd content in the scale of tens of nanometers, which allowed investigating the effects of concentration by simple sputtering of surface layers of the as-prepared samples.

\section{EXPERIMENTAL}

Several GaN films with the thickness of $3 \mu \mathrm{m}$ grown by MOVPE on $c$-plane sapphire substrates were subjected to ion implantation at room temperature using a flux of $\mathrm{Gd}$ ions with the energy of $200 \mathrm{keV}$, using doses in the range of $(1-10) \times 10^{16}$ atoms $\mathrm{cm}^{-2}$. Total amount of Gd ions actually detected in the samples was, however, an order of magnitude lower, which should be associated with sputtering effects occurring during the implantation. The annealing was performed in nitrogen atmosphere at $900{ }^{\circ} \mathrm{C}$ for $30 \mathrm{~min}$. A surface layer of about $20 \mathrm{~nm}$ of thickness was removed from a part of the implanted films by sputtering $\left(5 \mathrm{keV} \mathrm{Ar}^{+}\right.$ions,

\footnotetext{
a) Author to whom correspondence should be addressed. Electronic mail: hejtman@fzu.cz.
}

$\left.80^{\circ}\right)$. The composition of the films and the concentration profiles of $\mathrm{Gd}$ in $\mathrm{GaN}$ layers were measured using Rutherford backscattering (RBS). The x-ray photoemission was used for quantitative measurement of surface composition and the valence band structure study. The crystal structure of the films was analyzed by x-ray diffraction. Magnetic properties were studied on samples of the $5 \times 5 \mathrm{~mm}^{2}$ form using the SQUID magnetometer MPMS-5S (Quantum Design) in the field up to $5 \mathrm{~T}$ and the temperature range of $5-350 \mathrm{~K}$. High temperature investigations up to $750 \mathrm{~K}$ in the field of $1.9 \mathrm{~T}$ were made employing the magnetometer DSM10 (Manics) based on the determination of the force acting on the sample in a nonhomogeneous static magnetic field.

The $\mathrm{GGA}+U$ calculations of electronic structure (WIEN2K code) were carried out using two different supercells, $2 \times 2 \times 1$ and $2 \times 2 \times 2$, corresponding to compositions $\mathrm{Gd}_{0.125} \mathrm{Ga}_{0.875} \mathrm{~N}$ and $\mathrm{Gd}_{0.0625} \mathrm{Ga}_{0.9375} \mathrm{~N}$, respectively. Different values of the effective correlation potential $U_{\text {eff }}$ up to $8 \mathrm{eV}$ applied on Gd- $4 f$ states were selected. The energy cutoff $E_{\max }=300 \mathrm{eV}$ for plane wave expansion and the $k$ mesh equivalent to $2000 k$ points per Brillouin zone of parent $\mathrm{GaN}$ were used in all calculations.

\section{RESULTS}

No signs of loss of crystallinity or presence of secondary phases related to $\mathrm{Gd}$ were detected. The surface concentration of $\mathrm{Gd}$, measured by RBS, reached $x=0.02-0.07$ $\left.\left(0.8-3.0 \times 10^{21} \text { atoms cm}\right)^{-3}\right)$ and decreased to $x \sim 0.005$ $\left(0.2 \times 10^{21}\right.$ atoms $\left.\mathrm{cm}^{-3}\right)$ or less at the depths of $80 \mathrm{~nm}$. All samples showed similar characteristics as those found in MBE grown $\mathrm{Ga}_{1-x} \mathrm{Gd}_{x} \mathrm{~N}$ films, i.e., a small spontaneous magnetic moment, persisting well above room temperature, overwhelmed by the diamagnetic signal from the sapphire substrate.

The magnetic properties of our $\mathrm{Gd}$ implanted $\mathrm{GaN}$ films are illustrated in detail on a sample subjected to the dose of $5 \times 10^{16}$ atoms $\mathrm{cm}^{-2}$. The concentration profile, determined by RBS before and after the sputtering of the surface layer, is shown in Fig. 1. The integral amount of $\mathrm{Gd}$ ions in the as- 


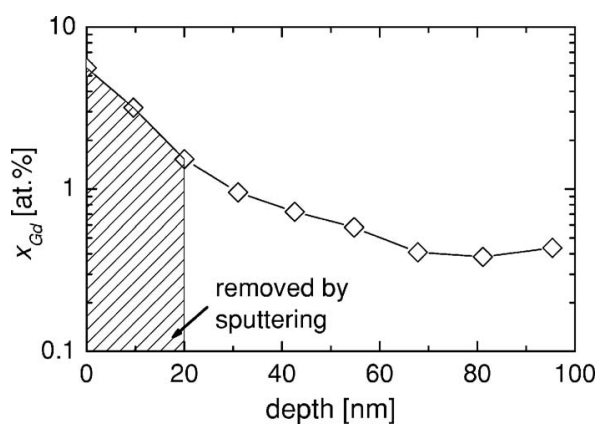

FIG. 1. Gd concentration profile in the ion implanted film GaN of $3 \mu \mathrm{m}$ thickness (total amounts) before and after the surface layer sputtering are 7.0 and $3.5 \times 10^{15} \mathrm{Gd}$ atoms $\mathrm{cm}^{-2}$, respectively.

prepared sample was calculated to $7 \times 10^{15}$ atoms $\mathrm{cm}^{-2}$ and about one-half of this amount was removed by sputtering. The surface $\mathrm{Gd}$ concentration was decreased from the original value $x=0.06\left(2.5 \times 10^{21}\right.$ atoms $\left.\mathrm{cm}^{-3}\right)$ to $x=0.01(0.4$ $\times 10^{21}$ atoms $\mathrm{cm}^{-3}$ ).

The isothermal magnetization data measured on specimens $0.5 \times 0.5 \mathrm{~cm}^{2}$ are presented in Fig. 2. Three main components can be separated: a strong diamagnetic signal from the substrate and two weak contributions from the Gd-doped GaN film, a paramagnetic (PM) component characterized by Brillouin $M(H)$ dependence at the lowest temperature and by a standard Curie-type $(1 / T)$ behavior at somewhat higher ones, as well as a small ferromagnetic (FM) component. The high temperature magnetic investigation shows that this FM component is nearly temperature independent and persists up to the temperature of $700 \mathrm{~K}$ (Fig. 3). It is of interest that the paramagnetic Curie-type contribution, clearly seen at low temperatures for the as-prepared sample (Fig. 2), is quite large pointing that practically all implanted $\mathrm{Gd}$ ions should contribute to it and play thus a passive role as magnetic impurities. This is consistent with the low value of the observed FM moment, $0.75 \times 10^{-5} \mathrm{emu} \mathrm{cm}^{-2}$. Considering the

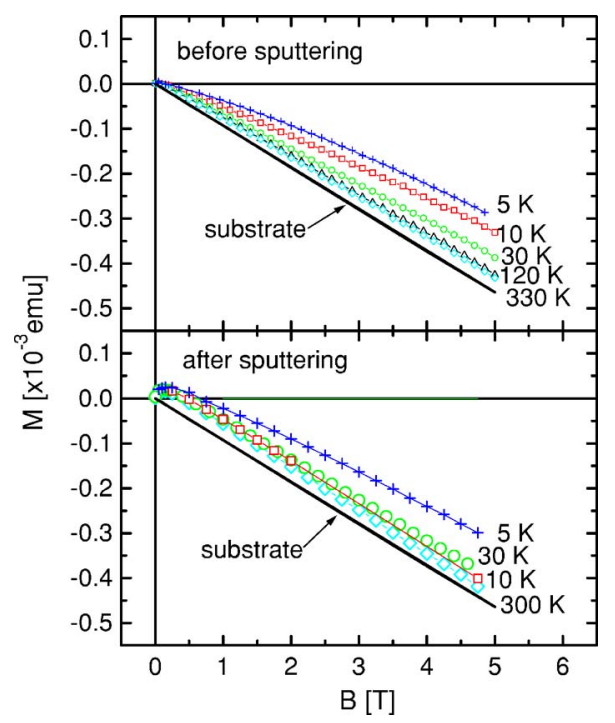

FIG. 2. (Color online) Isothermal magnetization curves $M(B)$ measured on sample $0.5 \times 0.5 \mathrm{~cm}^{2}$ before (upper graph) and after (lower graph) sputtering. The diamagnetic signal from the sapphire substrate is shown by thick line.

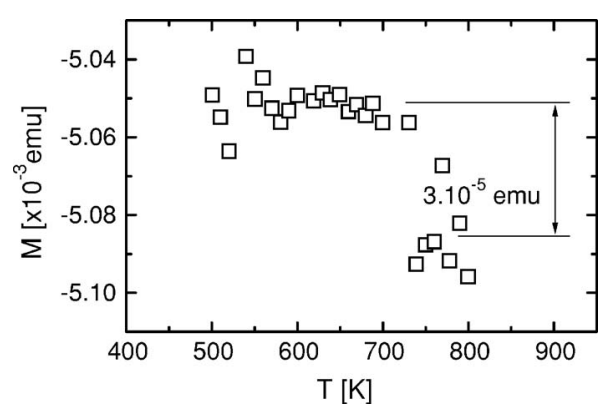

FIG. 3. The observed FM moment superposed on much stronger diamagnetic signal from substrate $(B=1.9 T)$. The step at $700 \mathrm{~K}$ defines $T_{C}$.

Gd doping rate of $7 \times 10^{15}$ atoms $\mathrm{cm}^{-2}$ in the as-prepared sample, this FM component corresponds to $0.7 \mu_{B} / \mathrm{Gd}$ atom, which suggests that only $\sim 10 \%$ of Gd ions participate in the FM phase. After the sputtering, the Curie-type behavior is suppressed considerably while the FM component increases to a value slightly higher than $2 \mu_{B} / \mathrm{Gd}$ atom, which corresponds to $1 / 4-1 / 3$ of Gd ions contributing to FM. Our observation thus clearly indicates that FM state should be attributed to a weakly Gd-doped inner GaN layers $(x \leqslant 0.01)$ while medium and highly doped outer layers are paramagnetic (see the concentration profile in Fig. 1).

The calculated electronic structure (Fig. 4) reveals the essential features of pure GaN, i.e., the $7.4 \mathrm{eV}$ broad valence band of $\mathrm{N} 2 p$ character and the conduction band formed predominantly by $\mathrm{Ga} 4 s$, both separated by a gap, whose width of $2 \mathrm{eV}$ is underestimated compared to experiment (a known deficiency of calculations based on density functional theory). The Gd doping is manifested by two sharp features: the filled spin-up and empty spin-down Gd- $4 f$ states split due to exchange $(J \sim 4.5 \mathrm{eV})$ and correlation $\left(U_{\text {eff }}\right)$ potential. For $U_{\text {eff }}=6.5 \mathrm{eV}$, which seems to be a reasonable value for lanthanides and has also been calculated by self-interaction corrected local density approximation (LDA), ${ }^{6}$ the spin-up band is positioned $-3.5 \mathrm{eV}$ below the valence band maximum (VBM), while the spin-down states are located $6 \mathrm{eV}$ above the conduction band minimum (CBM). The substitution of $\mathrm{Gd}$ for $\mathrm{Ga}$ is thus isovalent creating no extra electrons or holes. Nevertheless, the effective molecular field generated by $\mathrm{Gd}$ impurities induces a complete spin polarization at VBM (see the blowup in Fig. 4) and a small incomplete polarization at CBM as well. Hence, any additional doping will introduce spin polarized charge carriers. Lowering the

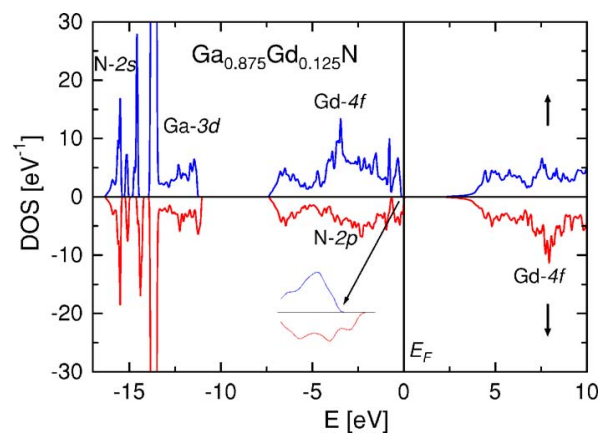

FIG. 4. (Color online) Electronic structure of $\mathrm{Gd}$ doped $\mathrm{GaN}$ $\left(\mathrm{Ga}_{0.875} \mathrm{Gd}_{0.125} \mathrm{~N}\right)$ calculated for $U_{\text {eff }}=6.5 \mathrm{eV}$. 
Gd concentration from $x=1 / 8$ to $x=1 / 16$ does not bring any qualitative changes, only the intensity of the respective features is reduced.

\section{DISCUSSION}

In summary, the present study confirms the existence of the FM phase in GaN films implanted by $\mathrm{Gd}$ ions. In contrary to previous reports, ${ }^{5}$ no indication for giant moments associated with $\mathrm{Gd}$ spins have been found. It appears that $\mathrm{Gd}$ ions at high doping levels contribute paramagnetically, while the FM phase seems to arise only at weak Gd dopings. In our sample, where doping levels range from 2.5 $\times 10^{21} \mathrm{Gd} \mathrm{cm}^{-3}$ for the surface layer to about $10^{20} \mathrm{Gd} \mathrm{cm}^{-3}$ for the inner parts, only minor portion of $\mathrm{Gd}$ ions belong to the FM phase.

The essential point that may help to understand the origin of the FM ordered state is the $n$-type semiconducting character of the virgin (undoped) GaN layers, brought about by a departure from ideal stoichiometry or by impurity defects. The shallow impurity levels are located in the gap just below CBM, or, if their concentration is increased, may form an impurity band merging with the conduction band and pinned to CBM.

Two different scenarios can be in principle envisaged. The first one is the donor impurity exchange scheme recently proposed for dilute magnetic semiconductors and based on a formation of bound magnetic polarons. ${ }^{7}$ However, for halffilled $4 f$ subshell of $\mathrm{Gd}$ as a magnetic impurity this model would require the presence of empty $4 f$ states in the vicinity of the shallow donor levels for the exchange interaction to become effective. This can hardly be the case here, even if one considers the real CBM shifted by $1.3 \mathrm{eV}$ higher than that obtained from GGA+U, Fig. 4. Second, the itinerant charge carriers, either those excited from the impurity levels to the conduction band or those forming an impurity band in the degenerate semiconductor limit, can mediate the magnetic interaction. This is the essence of RKKY mechanism which gives a FM exchange coupling between distant magnetic ions if the conduction band filling is low (small $k_{F}$ ). In its extended form RKKY takes into account the mean free path of spin polarized charge carriers. For higher Gd concentration the mean free path is expected to be shortened and, in agreement with present observation, an increasing part of $\mathrm{Gd}$ ions will not experience FM exchange interaction and appear in PM state.

\section{ACKNOWLEDGMENTS}

This work was supported by the Czech Science Foundation, grant No. GA104/06/0642 and the Ministry of Education of Czech Republic, Project No. MSM6046137302.

${ }^{1}$ T. Dietl, Phys. Status Solidi B 240, 433 (2003).

${ }^{2}$ L. Pérez, G. S. Lau, S. Dahar, O. Brandt, and K. H. Ploog, Phys. Rev. B 74, 195207 (2006).

${ }^{3}$ J. K. Hite, R. M. Frazier, R. P. Davies, G. T. Thaler, C. R. Abernathy, S. J. Pearton, J. M. Zavada, E. Brown, and U. Hömmerich, J. Electron. Mater. 36, 391 (2007).

${ }^{4}$ S. Y. Han, J. K. Hite, G. T. Thaler, R. M. Frazier, C. R. Abernathy, S. J. Pearton, H. K. Choi, W. O. Lee, Y. D. Park, and J. M. Zavada, Appl. Phys. Lett. 88, 042102 (2006).

${ }^{5}$ S. Dhar, T. Kammermeier, A. NeyL. Perez, K. PloogA. Melnikov, and A. D. Wieck, Appl. Phys. Lett. 89, 062503 (2006).

${ }^{6}$ A. Svane, N. E. Christensen, L. Petit, Z. Szotek, and W. M. Temmerman, Phys. Rev. B 74, 165204 (2006).

${ }^{7}$ J. M. D. Coey, M. Venkatesan, and C. B. Fitzgerald, Nano Lett. 4, 173 (2005) 